\title{
EL PROCESO DE INVESTIGACIÓN DENTRO DE LA FUNDACIÓN UNIVERSITARIA DE CIENCIAS DE LA SALUD
}

Marcela Gómez Suárez, MD*

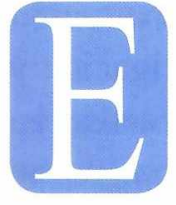

1 pasado mes de julio se llevó a cabo la jornada interfacultades de investigación con la presentación de los trabajos de pre y postgrado de los estudiantes de la institución, evento que sirve como cierre del proceso de formación investigativa que se desarrolla en conjunto entre las facultades y la División de Investigaciones. Enmarcado dentro de este evento se planteó la pregunta sobre ¿Cómo funciona la investigación dentro de la Fundación Universitaria de Ciencias de la Salud?

Para responderla lo primero en importancia para tener en cuenta es que la Fundación tiene antecedentes muy fuertes que apoyan el proceso de investigación: un hospital como San José que lleva 100 años formando profesionales de la salud partiendo de un grupo de profesores reconocidos en sus áreas de trabajo en ciencias básicas y clínicas, quienes han presentado el producto de su experiencia en diferentes ámbitos científicos; cuatro facultades conformadas y estructuradas, dirigidas a formar personal dedicado y reconocido por sus excelentes características profesionales, una revista institucional que lleva mas de 90 años publicando el resultado del conocimiento de la comunidad científica, una biblioteca y laboratorios que permiten motivar y aproximar a quienes tengan inquietudes científicas.

Desde el año 1997 se dieron los primeros esfuerzos para estructurar el proceso formal de investigación dentro de la Fundación, creando un comité a cargo del doctor William Rojas, el cual hizo el intento de reunir líneas y grupos de investigación basado en los intereses de los profesores. Aún a

\footnotetext{
Editorialista invitada. Médica y cirujana de la Pontificia Universidad Javeriana, especialista en salud ocupacional de la Escuela Colombiana de Medicina, Master en epidemiología clínica de la Pontificia Universidad Javeriana. Directora División de Investigaciones Fundación Universitaria de Ciencias de la Salud.
}

pesar que fue un gran esfuerzo, la investigación en este punto todavía se daba en forma aislada entre un pequeño grupo de docentes interesados en el tema, basada más en el recuento de su experiencia clínica y no se consideraba prioritaria la cultura de investigación interdisciplinaria.

En el año 2002, el Consejo Superior estableció dentro del organigrama de la institución una nueva dependencia, la División de Investigaciones a cargo de la doctora Martha Beatriz Montenegro quien fue su primera directora y le dió los lineamientos como unidad de apoyo a las facultades para desarrollar el proceso de formación investigativa y acompañamiento a docentes y estudiantes en la elaboración y conducción de trabajos de investigación.

Hoy en día la División de Investigaciones fundamenta su actividad formativa en que para poder realizar investigación de alto nivel hay que partir por generar una cultura básica de cuestionamiento crítico entre los estudiantes y profesores. Cuenta con un grupo multidisciplinario comprometido de profesionales con formación especializada en epidemiología y estadística quienes conducen la cátedra de metodología de investigación en pregrado para las cuatro facultades, la rotación obligatoria de tiempo protegido dirigida a los residentes de la facultad de medicina; asesoran y acompañan a los estudiantes de postgrado y a los profesores en la conceptualización, conducción y análisis de proyectos.

La División de Investigaciones participa en los comités de investigación de las diferentes facultades donde se discuten y aprueban los trabajos que van a ser realizados, publicados y presentados a nombre de la Fundación. Así mismo hace parte del comité de ética hospitalaria del hospital de San José. 
Además de esta labor académica y con miras a lograr la consolidación de líneas y grupos de investigación que representen la producción científica de la Fundación, la División está involucrada en la inscripción de estos grupos en instituciones como Colciencias, lo cual garantiza apoyo económico y reconocimiento científico nacional e internacional, así como la posibilidad para generar alianzas estratégicas con otras instituciones.

El camino recorrido hasta ahora se empieza a ver reflejado en el interés y participación de estudiantes $\mathrm{y}$ algunos profesores por investigar, asistir a eventos, publicar y presentar trabajos para la obtención de premios; pero el proceso aún es joven y falta consolidar y mejorar partes del mismo, se requiere mayor motivación por parte de la comunidad académica y un entendimiento de la importancia que tiene la investigación y el aprendizaje de su metodología para cualquier disciplina de la vida profesional. Debemos aceptar que las tendencias actuales nos obligan a dejar de reproducir las experiencias de otros y a desarrollar conocimiento nuevo y propio. Esta es la responsabilidad que tenemos como institución educativa con el país en materia de investigación y gracias al apoyo de las directivas de la Fundación intentamos cumplirla. Valga además este comentario para invitar a toda la comunidad académica a participar de este proceso vinculándose a las actividades desarrolladas desde la División de Investigaciones, con la certeza que encontrarán apoyo científico y económico que facilite el proceso. 